\title{
Imvume unolwazi ocwaningweni lokuvimbela igciwane lesandulela ngculaza
}

Population Council

Follow this and additional works at: https://knowledgecommons.popcouncil.org/departments_sbsr-hiv

Part of the Demography, Population, and Ecology Commons, Family, Life Course, and Society Commons, Gender and Sexuality Commons, International Public Health Commons, and the Medicine and Health Commons How does access to this work benefit you? Let us know!

\section{Recommended Citation}

"Imvume unolwazi ocwaningweni lokuvimbela igciwane lesandulela ngculaza." New York: Population Council, 2008. 
IMVUME UNOLWAZI OCWANINGWENI LOKUVIMBELA IGCIWANE LESANDULELA NGCULAZA

UMBIKO OPHUMA EMHLANGANWENI WOMHLABA 


\section{IMVUME UNOLWAZI OCWANINGWENI LOKUVIMBELA IGCIWANE LESANDULELA NGCULAZA}

UMBIKO OPHUMA EMHLANGANWENI WOMHLABA 
Copyright (C) 2008 The Population Council, Inc.

\section{(1) Population Council}

I-Population Council yenza ucwaningo emhlabeni ngobubanzi bawo ukwenza ngcono izinqubo, izinhlelo, nemikhiqizo ezihlokweni ezintathu: Igciwane lesandulela ngculaza neNgculaza; inhlupheko, ubulili, nentsha; kanye nempilo yokuzalana.

One Dag Hammarskjold Plaza

New York, NY 10017 USA

Ucingo: +1-212-339-0500

Isikhahlamezi: +1-212-755-6052

\section{Amazwi okubonga}

Umhlangano mayelana nemvume unolwazi ocwaningweni lokuvimbela igciwane lesandulela ngculaza, owawubanjwe kusukela ngomhlaka 16 kuze kube umhlaka 18 ku May 2005 edolobheni lase-NewYork, wawuhlanganiswe wahlelwa yikomidi elalino-Barbara Friedland, u-Martha Brady, no-Hillary Bracken (be-Population Council), u-Kathleen MacQueen (we-Family Health International), u-Cynthia Woodsong (we-Research Triangle Institute), no-Elizabeth McGrory (i-consultant). Umhlangano wawuphethwe i-Population Council ne-Family Health International futhi wawesekwa yi-United States Agency for International Development, i-International Partnership for Microbicides, Inc., National Institutes of Health, kanye ne-William and Flora Hewlett Foundation.

Ukuhumusha kwenziwe ngu: Duduzile Dlamini

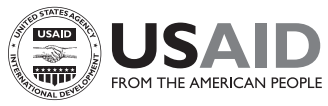

Lolulwazi lwenziwe lwayimpumelelo ngokwesekwa ngothando ngabantu baseMelika besebenzisa i-United States Agency for International Development (USAID). Okuqukethwe lapha kulolulwazi kuwumsebenzi we-Population Council iyodwa futhi akuvezi imibono ye-USAID noma uHulumeni wase-United States.

Noma iyiphi ingxenye yalolulwazi ingakopishwa noma ingashintshwa ukuze ihlangabezane nezinga lendawo ngaphandle kokuthola imvume kwi-Population Council, uma nje lezozingxenye ezikopishiwe zabiwa mahhala noma ngentengo (engenayo inzalo) nokuthi futhi umthombo ukhonjwe/ushiwo. Noma yikuphi ukukhiqiza okwenzelwa imali kudinga ukuthola imvume kuqala kwi-Population Council. I-Population Council ingakuthokozela ukuthola umfanekiso wanoma iziphi izinto lapho umbhalo usetshenziswe khona.

www.popcouncil.org/pdfs/ICWorkshopHighlightsZU.pdf 


\section{Okuqukethwe}

Isingeniso

Izinsiza zemvume unolwazi: Ukudlulisa imibono elukhuni

Izincwajana

Amavidiyo

Ukufundiswa kombambiqhaza ngendlela yokunikezelana encike kwikhompuyutha

Ukuchaza amgama alukhuni

Ukubheka ukuqonda phakathi kwababambiqhaza bocwaningo:

Yini abadinga ukuyazi ababambiqhaza?

Ukubaluleka kokuqeqesha

Isithasiselo 1: Indlela yokubuza imibuzo engenza ukuthi

izimpendulo ukwazi ukuzibala njengokuthi bangaki o"yebo" noma o "cha" nendlela yokubuza lapho obuzwayo eba nethuba lokuchaza kabanzi ngamazwi akhe (quantitative and qualitative approaches)

Isithasiselo 2: Ucwaningo lwe-HPTN 035: Uhla lokubheka ukuqondwa kwemvume unolwazi yokungeniswa (okucashuniwe) 


\section{Isingeniso}

G sikhathini esithuthuka ngokushesha socwaningo lokuvimbela igciwane lesandulela ngculaza, imvume unolwazi ibonakala njengendlela ebucayi yokwenza isikompilo lisebenze. Ekubeni ukuqinisekisa imvume unolwazi nokubamba iqhaza ngokuzikhethela okunye kwezinto ezibukhuni kakhulu ocwaningweni lwezempilo, ucwaningo lokuvimbela igciwane lesandulela ngculaza, okuningi kwako okwenziwa ezindaweni ezinezinsiza ezingaphelele, kudala izinkinga ezinkulu zokubhekeleka kwabantu.

- Ucwaningo olukhulu lokubheka ukusebenza ngempumelelo ludinga ukwenziwa ezindaweni ezinezinga eliphezulu legciwane lesandulela ngculaza.

- Ucwaningo ludinga amavolontiya aphilile, avamise ukungami kahle kwezomnotho nantekenteke kwinhlalakahle kanye nabasengcupheni enkulu yokutheleleka ngegciwane lesandulela ngculaza.

- Abacwaningi kumele baqinisekise ukungaziwa kokusebenza ngempumelelo komkhiqizo ohlolwayo ukuze ababambiqhaza bangazizwa benokuvikeleka okungamanga, noma ukungaqondi kahle ngezokwelapha, okungaholela ekunyukeni kokuziphatha ngendlela enobungozi (ukunciphisa ukusetshenziswa kwamakhondomu noma ukunyusa isibalo sophathina).

- Ucwaningo lokuvimbela igciwane lesandulela ngculaza/ingculaza luhlanganisa izinto ezizwelayo kakhulu: ukucwasa, ezocansi, kanye nodaba lwamandla oluphathelene nobulili.

- Ukudlulisela amatemu obuchwepheshe ocwaningo namagama angajwayelekile ngezilimi zasendaweni kungaba lukhuni.

Lezizinselelo zenziwe zaba zimbi kakhulu ezindaweni lapho izinga lokufunda liphansi, lapho abantu abasethubeni lokubamba iqhaza ocwaningweni bengaba nokuqonda okuncane mayelana nempilo yezocansi noma ucwaningo lwezempilo.

Ukubhekana nalezizinselelo, i-Population Council ne-Family Health International (FHI) benza umhlangano ukukhomba izindlela ezinempumelelo kwinqubo yemvume unolwazi ezingathathwa abacwaningi abenza ucwaningo lokuvimbela igciwane lesandulela ngculaza babelane ngazo. Lolulwazi lucashunwe embikweni ogcwele womhlangano, ${ }^{1}$ lubheka amathuluzi anokuthuthuka kanye namasu asetshenziswayo ukwenza imvume unolwazi ocwaningweni lokuvikela igciwane lesandulela ngculaza, futhi luhlanganisa izibonelo ezichaza kabanzi ezithathwe ocwaningweni oseluphelile nolusaqhubeka.

${ }^{1}$ C. Elizabeth McGrory, Barbara A. Friedland, Cynthia Woodsong, and Kathleen M. MacQueen. 2006. Informed Consent in HIV Prevention Trials: Report of an International Workshop. ("Imvume unolwazi ocwaningweni lokuvimbela igciwane lesandulela ngculaza: Umbiko ophuma emhlanganweni womhlaba.”) E-New York: Population Council and Family Health International. Utholakala ku: www.popcouncil.org/pdfs/ICWorkshop.pdf. 


\section{Amathuluzi emvume unolwazi: Ukudlulisela imibono elukhuni}

A bahloli ocwaningweni oluningi lwamanje lokuvimbela igciwane lesandulela ngculaza bahlola ngamathuluzi ahlukahlukene asezingeni lokwakha kangcono kanye nezindlela zokwenza ngcono ukuqonda kwamavolontiya amagama asocwaningweni kanye nezinqubo zocwaningo, kuhlanganisa izincwajana zocwaningo, amapheshana olwazi, ibhodi eligoqwayo elinamashadi okubhalela, I amavidiyo, izinsiza ezibonakalayo, kanye nendlela enokuxoxisana encike kwikhompuyutha yokufundisa umbambiqhaza. ${ }^{2}$

\section{Amasu okwakha izindlela kanye nezinsiza zemvume unolwazi}

- Ukwenza ucwaningo olunokuqaphela olwakhayo: ulwazi olungajulile, ukuhlola kuqala, nokujwayela izimo zasendaweni.

- Faka abasebenzi bocwaningo, amalunga ebhodi yabeluleki bomphakathi, abangaba ababambiqhaza, futhi, uma kungenzeka, ababambiqhaza bocwaningo oseludlule ekwakheni imibono, ekwakheni izindlela zokubhekana nocwaningo, kanye nokuhlola kuqala izinsiza.

- Sebenzisa amabhodi aluleka umphakathi kanye nezinye izinhlaka zasemphakathini ukuqinisekisa amagama abalulekile, njengokuzinikela, nokusiza ukuthuthukisa ulimi, amatemu, kanye nezindlela zokuchaza ezidlulisela amatemu ocwaningo ahambisana futhi azwakala kahle ngokwasendaweni.

- Sebenzisa izinsiza ngokuhlanganisa ekwakheni nasekuqinisaneni omunye nomunye ekwaziseni umphakathi nababambiqhaza ngolwazi olufanele kuyona yonke inqubo yocwaningo lwezempilo.

${ }^{2}$ Indlela yobuchwepheshe yokusebenzisana ingafaka i-ACASI-okuyi-audio computer-assisted self-"instruction" (indlela yokuzinika imiyalelo ehlanganisa ikhompuyutha kanye nokulalela) "noma ingxoxo oyibamba wena uqobo"-esetshenziselwa ukunikeza amavolontiya ocwaningo ulwazi noma indlela yengxoxo yokuthola izimpendulo mayelana nezihloko ezizwelayo. 


\section{Izincwajana}

T ncwajana ekhiqizwe kahle idlulisa ubuchwepheshe bocwaningo kanye nenhlonipho

1 kubabambiqhaza. Indlela yokuchaza esebenzisa imibala yemvelo ingabasiza ababambiqhaza ukuqonda nokugcina ulwazi. Izincwajana zocwaningo zingaba nenzuzo eku:

- nikezeni ababambiqhaza ulwazi jikelele noma olujulile mayelana nezinyathelo nezinqubo zocwaningo, kanye nokuchibiyela amafomu emvume unolwazi;

- chazeni nasekukhombiseni kabanzi amatemu alukhuni ocwaningo ngokusebenzisa imidwebo;

- phenduleni imibuzo ngezikhathi zokunikeza ulwazi;

- sizeni ukunxenxa uma ababambiqhaza bekhombisa izihlobo noma abangani izincwajana.

Ukusebenza kwamathuluzi emvume unolwazi: Ucwaningo lwesigaba sesithathu lwe-Carraguard ${ }^{\circledR}$ ye-Population Council

Incwajana yocwaningo lwemayikhrobhisayidi lwesigaba sesithathu lwe-Carraguard ${ }^{\circledR}$ ye-Population Council ${ }^{3}$ yachibiyela imvume unolwazi. Ngesikhathi sokwenza ukuhlola ngaphambi kokuba ucwaningo luqale, ababambiqhaza basho imibono mayelana nemifanekiso, umbala, kanye nokubaseqophelweni jikelele bencwajana, okwaholela ekushintshweni kwesakhiwo sencwajana (bheka ibhokisi).

\section{Ukusebenza kwamathuluzi emvume unolwazi: Ucwaningo lwemayikhrobhisayidi} i-HPTN 035

Incwajana yocwaningo lwemayikhrobhisayidi lwe-HIV Prevention Trials Network (HPTN) $035^{4}$ isebenzisa imidwebo yomlingisi obizwa ngokuthi uSerena ukuchaza amagama amaningi ocwaningo kanye nezinqubo. Lolucwaningo olwalwenziwa ezizindeni eziningi lwalwenziwa emazweni amaningana, ngakhoke izinsiza zahlolwa ngaphambi kokuqala kocwaningo futhi zenziwa zazwana nendawo ngayinye. Nakuba uSerena enziwa ngenhloso ukuthi ajwayeleke endaweni yabesifazane ababenxenxelwa ucwaningo, ukuhlola kuqala kwaholela ekuthini uSerena adwetshwe kabusha ukuze abukeke njengomuntu wesimanje, ohehayo, nophila impilo ephezulu. Futhi, omunye umdwebo kaSerena wahunyushwa abaningi ababengaba ababambiqhaza njengomuntu okhathazekile, ngakhoke wase edwetshwa kabusha (bheka ibhokisi).

\footnotetext{
${ }^{3}$ Ucwaningo lwesigaba sesithathu lokuhlola ukuphepha nokusebenza ngempumelelo kwemayikrobhisayidi i-Carraguard ukuvimbela ukutheleleka ngegciwane lesandulela ngculaza ebantwini besifazane lwalwenziwa ezizindeni ezintathu e-South Africa phakathi kuka Mashi ngo 2004 no Mashi 2007. www.popcouncil.org/projects/MIC_CarraClinicalStudies3.html. Yahlolwa ngo January 17, 2008.

${ }^{4}$ Ucwaningo lwamaqembu amane ofakwa kuwona ngokwethuba i-HPTN 035 ucwaningo lwesigaba 2/2b lokuphepha nokusebenza ngempumelelo kwamamayikhrobisayidi azinhlobo zimbili kanye namaqembu amabili okuqhathanisa olwangenisa abantu besifazane abayizinkulungwane ezintathu namakhulu amabili namashumi amabili $(3,220)$ ezizindeni eziyisishiyagalombili emazweni amahlanu. www.hptn.org/research_studies/hptn035.asp; www.mtnstopshiv.org/node/62. Yahlolwa ngo January 17, 2008.
} 
Ukusetshenziswa kwencwajana ye-Carraguard yesigaba sesibili esigabeni sesithathu
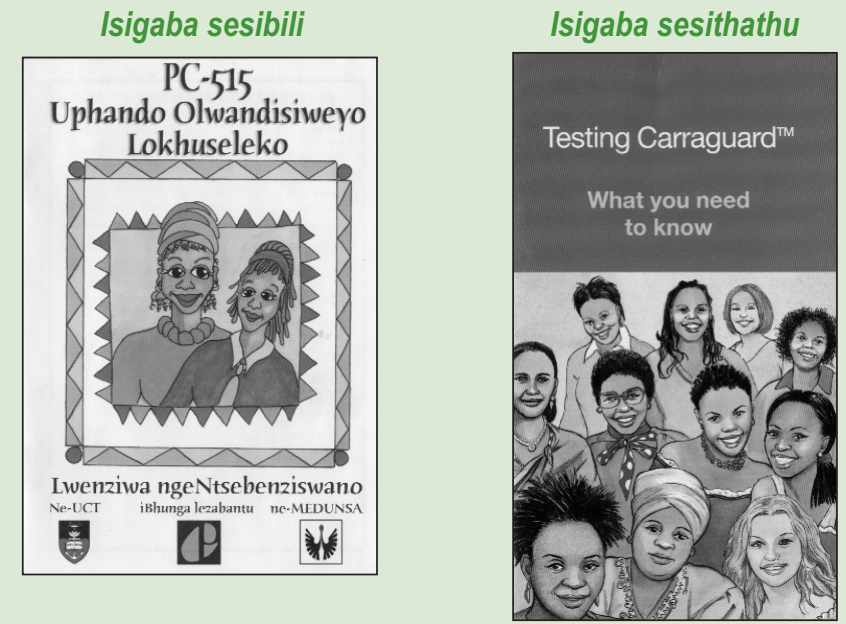

Ababambiqhaza bocwaningo ocwaningweni Iwe-Carraguard Iwesigaba sesibili bancoma ukuthi ukuchaza izithombe zencwajana yolwazi (esinxeleni) kube okungenzeka. Kwenziwa ushintsho kwincwajana ye-Carraguard isigaba sesithathu (Isikudla).

\section{Ukuthuthukiswa kuka "Serena” kwi HPTN 035}
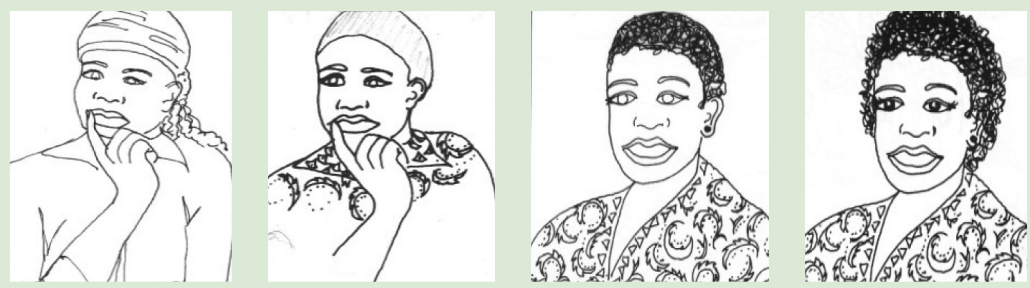

Ekuhloleni ngaphambi kocwaningo, abesifazane basiza ukwakha uSerena, osetshenziswe ezincwajaneni zocwaningo. (Artwork by Denise Todloski) 


\section{Amavidiyo}

A mavidiyo anikeza ithuba lokwenza ukunxenxa nezinqubo zemvume unolwazi A kube lula, ikakhulukazi ocwaningweni olukhulu. Amavidiyo angakhuthaza kakhulu kunokubhaliwe, angaba ithuluzi elingasiza ngokukhethekile ezindaweni ezinezinga lemfundo eliphansi, futhi angasiza ekwenzeni ukunikeza ulwazi locwaningo kube sezingeni elifanayo nasekunciphiseni umthwalo kubasebenzi bocwaningo.

\section{Izinselelo zobuchwepheshe bamavidiyo zihlanganisa:}

- Inqubo yokuthuthukisa ebukhuni idinga isikhathi eside, okungenza ukuthi kube lukhuni ukufaka ushintsho kuhlelo locwaningo.

- Ukukhombisa ividiyo kungangenzeke kuzo zonke izindawo zokunxenxa ngenxa yokuthi ukuletha izimpahla ezibizayo ezizindeni ezisendaweni kungenzeka kube nzima noma kube yingozi.

- Ukuheha intshisekelo yalabo abangaba ababambiqhaza emaklinikhi amatasatasa kungaba lukhuni.

Ukusebenza kwamathuluzi emvume unolwazi: Ucwaningo lwe-Latex diaphragm lwakwa MIRA lokuvikela ukutheleleka ngegciwane lesandulela ngculaza kubantu besifazane

Abasebenzi bocwaningo lweMIRA ${ }^{5}$ bathola ukuthi ababambiqhaza babekhombisa ukukhathala yizinto ezibhaliwe noma ukuzwa abasebenzi basocwaningweni bethula ulwazi; amavolontiya akhombisa ukuthi amavidiyo angawasiza ukubagcina bekhuthazekile.

- Odokotela namanesi, amalunga omphakathi, nethimba labacwaningi babeyingxenye ekwakhiweni kwevidiyo.

- Ababambiqhaza bocwaningo bazwakalisa ukukwamukela ukuvela kuka sihlalo we-ethics committee evidiyweni, okwa:

-siza ukunikeza ubuso bangempela ekubuyekezweni kwendlela ekahle yokuziphatha "ethical review";

-thumela umyalezo wokuthi ababambiqhaza bathathelwa phezulu;

-dlulisa ubuchwepheshe bocwaningo.

\footnotetext{
${ }^{5}$ Ucwaningo lwe-MIRA (Methods for Improving Reproductive Health in Africa) lwenziwa eThekwini naseGoli, e-South Africa, nase Harare, nase Zimbabwe.

www.cervicalbarriers.org/documents/MIRA_Results_and_Publications_aug07_final_pdf.pdf.

Yahlolwa ngo January 17, 2008.
} 
Ukusebenza kwamathuluzi emvume unolwazi: Ucwaningo lwesigaba sesithathu lwe-Carraguard ye-Population Council

Ukwenza ngcono izindlela zokwenza izinto ocwaningweni lwesigaba sesithathu lokubheka ukusebenza ngempumelelo kwe-Carraguard eSouth Africa, i-Population Council yenza izingxoxo namaqembu kanye nokubuzwa kwemibuzo okujulile kubabambiqhaza bocwaningo lwesigaba sesibili kanye nabasebenzi. ${ }^{6}$ I-Council yayifuna ukuthola indlela yokuchaza amagama abukhuni nokuthola imibono yokunxenxa kanye nenqubo yemvume unolwazi yocwaningo lwesigaba sesithathu. I-Population Council yenza ividiyo, ${ }^{7}$ iyisusela ezimpendulweni, yokucacisa amagama angajwayelelekile nokwethula ulwazi oludingekayo ngendlela ekwenza unake.

- Izindatshana ezimfishane zazisetshenziswa ukudlulisela amagama abukhuni kanye nezingqikithi njengokufakwa eqenjini ngendlela yokwethuba (engenalo uhlelo), ukungazi ngeqembu okulona kongumbambiqhaza nakongumsebenzi wocwaningo (double blinding), ukubamba iqhaza ngokuzikhethela, ukuxhumana nophathina, ukuhlolwa kwezitho zangasese (inqubo engajwayelekile eningini labesifazane emiphakathini yocwaningo), nokuhlolwa kwegciwane lesandulela ngculaza.

- Umeluleki wezemfundo wasiza ukwakha umbhalo, nomlingisi wesifazane owaziwa kakhulu wavela njengomxoxi.

- Izigaba zevidiyo zahlolwa kuqala zase sisetshenziswa ngokulandela izingxoxo zamaqembu nabesifazane emiphakathini yocwaningo kanye namalunga ebhodi yabeluleki bomphakathi.

- Imibiko engahloliwe iveza ukuthi abasebenzi bocwaningo nababambiqhaza bocwaningo bayithola ifundisa ividiyo kahle futhi ithakaseleka; abesifazane bakuncoma ukubona kuqala ababezobhekana nakho ocwaningweni.

\footnotetext{
${ }^{6}$ A De Kock, M. Marumo, B. Friedland. 2005. "Carraguard Phase 3 Trial: Developing and implementing a video." Ukwethulwa emhlanganweni womhlaba we-Population Council/Family Health International mayelana nemvume unolwazi ocwaningweni lokuvimbela igciwane lesandulela ngculaza, e-New York kusukela ngomhlaka 16 kuze kube umhlaka 18 ku May.

7 “Testing Carraguard ${ }^{\mathrm{TM}}$. What you Need to Know." Ividiyo. Johannesburg: Population Council, 2004. Yashicilelwa ngabakwa-Pandamonium Productions. www.popcouncil.org/media/saclips/videolink.html. Yahlolwa 17 January 2008.
} 


\section{Ukufundiswa kombambiqhaza ngendlela enokuxoxisana okuncike kwikhompuyutha}

Ukusebenza kwamathuluzi emvume unolwazi: Ucwaningo lwePrEP i-BOTUSA projekthi

Ucwaningo lwePrEP i-BOTUSA projekthi ${ }^{8}$ lwethula uhlelo lokusebenzisa indlela yokunikezelana neyokuzifundisa kusetshenziswa ikhompuyutha njengokugcizelela emva kwenqubo yokunikeza ulwazi olujulile olusiza ukunika imvume yobuso nobuso futhi ngaphambi kokwenziwa kokuhlolwa kokuqonda. Ulwazi lwesimanje lusebenzisa ikhompuyutha ene-screen esithintwayo nezinto zokulalela, futhi ulwazi mayelana nocwaningo ludluliswa ngendlela yokwethulwa kwezithombe nomsindo kuphelekezelwa wumxoxi, ababambiqhaza banakho ukuzikhethela ulimi.

${ }^{8} \mathrm{~J}$ Ucwaningo lwe-PrEP lwase Botswana, ucwaningo lokuhlola ukuphepha nokusebenza ngempumelelo kwendlela yokuvimbela ukutheleleka esetshenziswa ngaphambi kokuba ube sengozini yokutheleleka ekunciphiseni ukutheleleka ngegciwane lesandulela ngculaza ebantwini abasha bobulili obungafani, lwenziwa iBOTUSA Project-inhlanganisela ka Hulumeni waseBotswana ne-US Centers for Disease Control and Prevention (CDC). www.cdc.gov/hiv/resources/factsheets/prep.htm. Yahlolwa ngo January 17, 2008. 


\section{Ukuchaza amagama abukhuni}

A magama kanye namatemu ocwaningo abukhuni impela ukuwadlulisela kumavoA lontiya ocwaningo ezindaweni ngokwehlukahlukana kanye nangezilimi ezihlukahlukene ngendlela eqinisekisa ukuqonda. Abasebenzi bocwaningo basebenzisa izindlela ezithuthukile ukunikeza ulwazi kanye nokuchaza amagama abukhuni ngokusebenzisa izinsiza kufundisa nokusebenzisa izinsiza kunikwa ulwazi lwemvume lulezozindawo.,10

Izibonelo zezindlela ezinokuthuthuka zokuchaza amagama abukhuni ezaxoxwa emhlanganweni zihlanganisa:

Ukufakwa eqenjini ngokwethuba (okungenalo uhlelo): Inqubo yokuhlukanisa ababambiqhaza ngamaqembu okwelashwa ngokubafaka ngokwethuba, okusetshenziselwa ukususa ukuchema ngokokukhetha kwabacwaningi noma ababambiqhaza, ukuqinisekisa ukuthi ukuhlaziya ngokwezibalo kwemininingwane yocwaningo kuzoba ngokusemthethweni, nokwakha amaqembu anezinto ezifanayo ezingaba nomthelela kumphumela. Ukufakwa eqenjini ngokwethuba yitemu elibukhuni locwaningo elingaba yinto engajawayelekile kulabo abangabamba iqhaza futhi kubukhuni ukulichaza njalo njalo ezindaweni ezihlukahlukene:

- Ukufakwa eqenjini ngokwethuba kuvamise ukuchazwa ngokusebenzisa imifanekiso ehlobene nokwenzeka ngethuba, njengomshini welotho noma ukugingqa idayisi (bheka ibhokisi).

- Umfanekiso wekhompuyutha ungenza iphuzu lokuthi uhlelo lwekhompuyutha, hhayi umuntu, yilona olufaka umbambiqhaza eqenjini lomuthi ohlolwayo noma lokuqhathanisa.

\section{Ukufakwa eqenjini ngokwethuba}
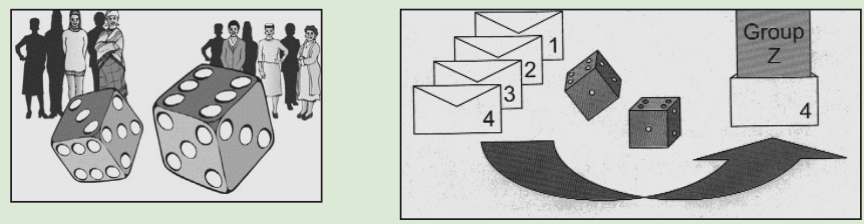

Imifanekiso yezinto ezihlobene nombono wethuba - ukugingqa idayisi- yasetshenziswa ukuchazela ababambiqhaza ukuthi bakhethwa ngokungenalo uhlelo ukufakwa eqenjini locwaningo lomkhiqizo ohlolwayo nakwelomkhiqizo ongenamuthi. Esinxeleni, izincazelo ezithathwe ocwaningweni lweCarraguard Iwesigaba sesithathu; esikudleni, okuthathwe ocwaningweni IweBOTUSA PrEP.

\footnotetext{
${ }^{9}$ H. Bracken. 2005. "Communicting complex concepts." Ukwethulwa emhlanganweni womhlaba we-Population Council/Family Health International mayelana nemvume unolwazi ocwaningweni lokuvimbela igciwane lesandulela ngculaza, e-New York kusukela ngomhlaka 16 kuze kube umhlaka 18 ku May.

${ }^{10}$ Ngoba ukusebenza ngempumelelo kwalezindlela zokudlulisela ulwazi kubabambiqhaza bocwaningo akukakahlolwa ngendlela ehleliwe, kumele kuthathwe njengeziphakamiso kunokuthi "okungcono okungenziwa."
} 
Umkhiqizo ongenamuthi: Izincazelo zigcizelela ukuthi umkhiqizo wocwaningo kanye nomkhiqizo ongenamuthi kufana ncamashi ngaphandle kokuthi umkhiqizo ongenamuthi:

- Awu "sebenzi," noma "awunaso [isithako esisebenzayo] kuwona"

- "Okukodwa kune [sithako esisebenzayo] okunye akunaso"

- Kuwu "ketshezi lokuqhathanisa" (bheka ibhokisi)

Ezinye izindlela zokuchaza umkhiqizo ongenamuthi zihlanganisa izifaniso zemifanekiso okufanele isuselwe ezimweni zasendaweni futhi ihlolwe ukuqinisekisa ukuthi isibonelo sifanelekile:

- Isiphuzo esinothiswe ngamavithamini noma ubisi

- Ubucwebe okungebona obangempela buqhathaniswa namatshe angempela asetshenziswa ebucwebeni

- Ama-cola mbumbulu eqhathaniswa ne-Coke ${ }^{\mathrm{TM}}$ yangempela

Isimo lapho ophathina ababili betholakala benezimo ezingafani zegciwane lesandulela ngculaza (Serodiscordance): Kungaba lukhuni impela ukuchaza ukuthi uphathina oyedwa angatheleleka ngegciwane lesandulela ngculaza omunye angatheleleki. Ukuqguqguzela izithandani ukuthi zihlolelwe igciwane lesandulela ngculaza, ucwaningo lwe-HSV/HIV olwenziwa yi-Partners in Prevention ${ }^{11}$ lusebenzisa umfanekiso wesihlahla se-baobab esihlaselwe umuhlwa (bheka ibhokisi). Umuntu angeke akwazi ukubona ekude ukuthi isiphi isihlahla esinomuhlwa noma ngeke akwazi ukubona ukuthi imuphi uphathina otheleleke ngegciwane lesandulela ngculaza ngaphandle kokuhlola. Isifaniso sesihlahla se-baobab singafaneleka kwezinye izindawo kodwa kwezinye ngeke, lokhu kuchaza kabanzi ukubaluleka kokuhlola kuqala ngokuhlobanisa imifanekiso nolimi endaweni ngayinye ukuqinisekisa ukuthi kudlulisa umyalezo oyiwonawona futhi kwenza ngcono ukuqonda.

Izinto ezizwelayo: Ezocansi nezinye izinto ezizwelayo kubhekwana nazo ngemifanekiso ecacile noma ekwazi ukwakha isithombe emqondweni (bheka ibhokisi):

- Ezinye izinsiza zisebenzisa imidwebo enjengezicathulo noma izingubo (ngaphandle kokuveza abantu) ukuveza ukuya ocansini.

- Ezinye zisebenzisa umdwebo ocace kakhulu wendoda nomuntu wesifazane benqunu belele ndawonye.

- Ngokufanayo, ukuhlolwa kwezitho zangasese kungakhonjiswa ngendlela ecacile noma imifanekiso engajulile kakhulu.

- Olunye ucwaningo lufaka imifanekiso yokuchazwa kokuhlolwa kwezitho zangasese kwizinsiza ezisetshenziswa esizindeni seklinikhi kuphela kodwa hhayi ezintweni ababambiqhaza abangaya nazo emakhaya, ukubhekana nokukhathazeka kokuthi ukuveza izithombe ezicacile ezintweni ezibhaliwe kungabonwa ngabanye abantu futhi kungadala ukucwaswa kwocwaningo noma ababambiqhaza.

\footnotetext{
${ }^{11}$ Ucwaningo lwesigaba sesithathu lwe-acyclovir lokucindezela i-HSV-2 phakathi kwezithandani ezinesimo segciwane lesandulela ngculaza esingafani, olwenziwa ezizindeni eziningi e-Africa lwenziwa i-Partners in Prevention Project (University of Washington). http://clinicaltrials.gov/show/NCT00194519; http://depts.washington.edu/hsvhiv/public/index.html. Yahlolwa ngo January 17, 2008.
} 


\section{Umkhiqizo ongenamuthi}

\section{I-Carraguard \\ kuhlanganisa \\ namakhondomu}

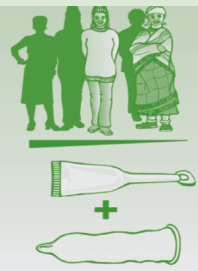

Uketshezi lokuqhathanisa kuhlanganisa namakhondomu

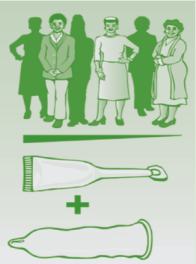

Ukusetshenziswa kwezithombe ezingabantu evidiyweni yocwaningo Iwesigaba sesithathu IweCarraguard kwagcizelela ukuthi umkhiqizo kanye nomkhiqizo ongenamuthi kubukeka ngendlela efanayo futhi kusetshenziswa ngendlela efanayo.

\section{Ukuhlolwa kwezitho zangasese}
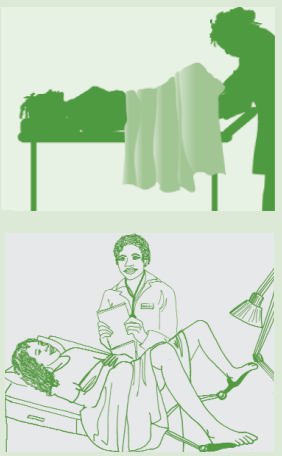

Abanye ababambiqhaza baveza ukukhathazeka ngokuthi ukucacisa kakhulu kwizinsiza umuntu angazithatha aziyise ekhaya noma zibonwe abanye abantu kungadala ukucwaswa kocwaningo noma kwababambighaza. Isithombe esiphezulu sithathwe ocwaningweni IweBOTUSA i-PrEP, esisezansi sithathwe kwi-HPTN.

Isimo lapho ophathina ababili betholakala benezimo ezingafani zegciwane lesandulela ngculaza (Serodiscordance)
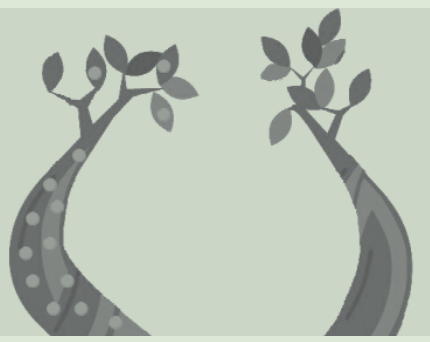

Izihlahla ezimbili ze-baobab zibukeka zifana nakuba esinye sihlaselwe umuhlwa ongonakalisa esisodwa noma zombili izihlahla. Lencanzelo isetshenziswa njengesifaniso segciwane lesandulela ngculaza, engahlasela oyedwa noma bobabili ophathina kodwa ayitholakali ngaphande kokuhlola. Imifanekiso ithathwe ocwaningweni olubizwa ngePartners in Prevention HSV/HIV.

\section{Okuphathelene nokuya ocansini}

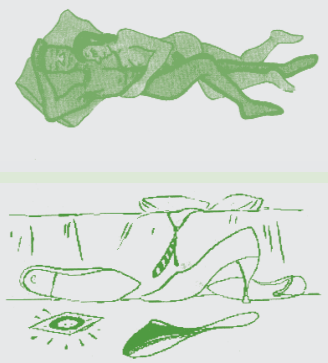

Ngokuncika esizindeni socwaningo, izinsiza ocwaningweni oluhlukene Iwe-Carraguard zasebenzisa imfanekiso ecacile (phezulu i-South Africa) noma ekwazi ukwakha isithombe emqondweni (ezansi i-Thailand) ukuveza ukuya ocansini. 


\section{Ukuhlolisisa ukuqonda phakathi kwababambiqhaza: Yini abadinga ukuyazi ababambiqhaza?}

T jengengxenye yokwenza ngcono imvume unolwazi, abasebenzi abaningi 1 bocwaningo bazama kanzima ukwazi ukuthi ungayikala kanjani indlela ababambiqhaza abaqonda ngayo izindaba zocwaningo. Abacwaningi abahlela ukuhlolwa kwemvume unolwazi babhekana nokukhetha phakathi kokuthi basebenzise indlela yokubuza imibuzo lapho umuntu ephendula khona ngamazwi akhe nendlela yokubuza imibuzo lapho umuntu ephendula ngokuthi 'iqiniso noma amanga' noma anikezwe uhla lwezimpendulo azokhetha kulona (quantative and qualitative approaches) (bheka uIsithasiselo 1).

- Ngabe ukuqondwa kwamagama athile nezinqubo zocwaningo kungathathwa njengokunesidingo, kangangokuba amavolontiya kumele akhombise ukuqonda kwawo ngaphambi kokuba avunyelwe ukungeniswa?

- Yiziphi izindlela okufanele zisetshenziswe ukuhlolisisa ukuqonda?

Nakuba inhloso yemvume unolwazi kungukuqonda okugcwele kwevolontiya zonke izidingo zocwaningo, ukuthola lelizinga lokuqonda kungenzeka kungahlali kwenzeka. Abacwaningi abaningi bacabanga ngezinto eziningi ezibucayi ukuthi amavolontiya ocwaningo aziqonde:

- Inhloso yocwaningo.

- Indlela ucwaningo oluhlelwe ngayo ukuze lukwazi ukuphendula imibuzo engakaze iphendulwe ngaphambilini.

- Ukungabi naso isiqiniseko sokusebenza ngempumelelo komkhiqizo ohlolwayo ukuvimbela ukutheleleka ngegciwane lesandulela ngculaza.

- Isidingo sokuqhubeka nezinye izindlela zokunciphisa ingcuphe yokutheleleka ngesikhathi sokubamba iqhaza ocwaningweni.

- Amatemu anjengokufakwa eqenjini ngokwethuba, ukungalazi iqembu okulona, nangemikhiqizo engenamuthi.

- Izinqubo zocwaningo, ubungozi, izinzuzo, ukuzikhethela nobumfihlo.

Bheka uIsithasiselo 2 ngokwesibonelo samathuluzi eHPTN okuhlolisisa ukuqonda. 


\section{Ukubaluleka kokuqeqeshwa}

T gisho nezinsiza ezingcono, amathuluzi, nokuhlolisisa ukuqonda akunalo usizo 1 oluphelele uma abasebenzi bocwaningo bengakutholi ukuqeqeshwa nokwesekwa ekukusebenziseni njalo nangendlela okuyiyona. Ukwakha nokusebenzisa izindlela zokudlulisa izinto ezibukhuni nokwenza ngcono imvume unolwazi, kanye nokuhlola ukusebenza kwazo ngempumelelo, kungabiza kakhulu futhi kudinga amandla engcebo nokuzimisela kwabaxhasi bocwaningo, abanikelayo, kanye namathimba ocwaningo. Amandla engcebo anele kudingeka afakwe kuhlelo lwezimali zocwaningo ukuqinisekisa ukuqeqesha okuqhubekayo kanye nokuhlolisisa.

\section{Isiphetho}

A bacwaningi kanye nabasebenzi ocwaningweni kuzona zonke izindawo kumele ukuqonda kwababambiqhaza kanye nokwenza ngcono imvume unolwazi. Ukwengeza, ekwakheni ubufakazi bokonga ngenhloso yokwenza inzuzo eqhubekayo, ukushintshisana okunje futhi kungaba nomthelela ekuxazululeni izingqinamba ezijwayelekile, ezinqumeni mayelana nokuthi iluphi uhlobo lokusakaza noma amasu azosetshenziswa, ekuqeqesheni, ezindleleni ezikhethekile zokudlulisa amatemu abukhuni. 


\section{Isithasiselo 1: Indlela yokubuza imibuzo engenza ukuthi}

izimpendulo ukwazi ukuzibala njengokuthi bangaki o"yebo" noma o "cha" nendlela yokubuza lapho obuzwayo eba nethuba lokuchaza kabanzi ngamazwi akhe (quantitative and qualitative approaches)

\begin{tabular}{|c|c|c|}
\hline & Amandla & Ubuthakathaka \\
\hline $\begin{array}{l}\text { Indlela yokubuza } \\
\text { edinga } \\
\text { impendulo } \\
\text { eyodwa } \\
\text { (indlela } \\
\text { engavulelekile, } \\
\text { ukuphendula } \\
\text { ngokuthi } \\
\text { amanga/ } \\
\text { iqiniso, } \\
\text { ukunikezwa } \\
\text { izimpendulo } \\
\text { ongakhetha } \\
\text { kuzona) }\end{array}$ & $\begin{array}{l}\text { - Ingcono ekufuneni } \\
\text { ukuthola impendulo } \\
\text { olwazini oluthile, } \\
\text { izimpendulo zegama } \\
\text { elilodwa, noma ukubheka } \\
\text { amaqiniso } \\
\text { - Ilula ukwenziwa } \\
\text { - Ukuhlolisiswa kwayo } \\
\text { akuchemile } \\
\text { - Idinga ukuqeqeshwa } \\
\text { kwabasebenzi okuncane. }\end{array}$ & $\begin{array}{l}\text { - Ayenabile ekuhloleni ukuqondwa kwatemu } \\
\text { abukhuni } \\
\text { - Ingadala ukukhohliseka ngokuthi umbambiq- } \\
\text { haza uzwile, ube ukala kuphela ukukwazi } \\
\text { ukuphinda ulwazi kwesikhathi esifishane } \\
\text { - Ukukhetha amagama/amatemu afanelekile } \\
\text { kubukhuni ocwaningweni lwezizinda eziningi/ } \\
\text { nezilimi eziningi } \\
\text { - Imibuzo ephendulwa ngokuthi amanga/iqiniso } \\
\text { noma enikeza izimpendulo ozokhetha kuzona } \\
\text { ihlanganisa ukusho okungekhona okungase } \\
\text { kudidise amavolontiya } \\
\text { - Imibuzo ephendulwa ngokuthi amanga/iqiniso } \\
\text { ivamise ukutshekela ngaseqinisweni } \\
\text { - Indlela yokuphendula ngokuthi amanga/ } \\
\text { iqiniso ayijwayelekile ezindaweni eziningi, } \\
\text { ivolontiya lingakubona kungukudelela ukusho } \\
\text { ukuthi into eshiwo umeluleki ayilona iqiniso }\end{array}$ \\
\hline $\begin{array}{l}\text { Indlela yokubuza } \\
\text { evulelekile } \\
\text { (Imibuzo } \\
\text { uyakwazi } \\
\text { ukuyiphendula } \\
\text { wenabe) }\end{array}$ & $\begin{array}{l}\text { - Yagqugquzela abantu } \\
\text { ukuthi basho okuningi, } \\
\text { babuze imibuzo, noku- } \\
\text { veza ngokucacile ukuthi } \\
\text { ulwazi luqondisisekile } \\
\text { - Ivumela umbambiqhaza } \\
\text { ukuthi aphendule ngamazwi } \\
\text { akhe kwinkulumo mpen- } \\
\text { dulwano ehambisana } \\
\text { nemvume unolwazi } \\
\text { - Abasebenzi bangakwazi } \\
\text { ukuthola ukuthi umbam- } \\
\text { biqhaza angakwazi } \\
\text { ukusebenzisa ulwazi } \\
\text { ukunquma ukuthi } \\
\text { abambe iqhaza ocwan- } \\
\text { ingweni nokulandela } \\
\text { ihlelo locwaningo }\end{array}$ & $\begin{array}{l}\text { - Ukuhlolisisa kuba nakho ukuchema; idinga } \\
\text { ukwahlulela kwabasebenzi okuseqophelweni } \\
\text { eliphezulu mayelana nokuqonda } \\
\text { kwevolontiya } \\
\text { - Idinga ukuqeqeshwa kwabasebenzi } \\
\text { okweluliwe nokwesekwa okuqhubekayo } \\
\text { - Idinga isikhathi esiningi sabasebenzi ukuze } \\
\text { yenziwe } \\
\text { - Inombolo yabantu okuhloswe ukuba } \\
\text { bangeniswe ingadala ukuthi abasebenzi } \\
\text { bangabi nawo umdlandla wokuthola ukuthi } \\
\text { ivolontiya aliluqondi kahle ulwazi ukuze } \\
\text { lingeniswe }\end{array}$ \\
\hline
\end{tabular}


